Division sponsored a symposium on the subject in California in December 1956. The reports presented at the symposium have now been collected into a printed volume, in order to make the information contained in them more generally available.

Because of its aims, the book does not delve deeply into the details and difficulties of the subject, but simply indicates in general terms the problems encountered and the progress so far made towards solving them.

The topics discussed were various. On the theoretical side these included scaling laws (Kantrowitz and Petschek, Landshoff), dynamo theories of cosmical magnetic fields (Elsasser, Hoyle), the penetration of shock waves into a magnetic field (Burgers), the dynamics of a pinched discharge just after onset (Rosenbluth), and hydromagnetic wave guides (Newcomb). The experiments described were chiefly concerned with shock waves, especially those driven by magnetic stresses (Kolb, Kash, Petschek), though shock waves suddenly passing into a magnetic region were also discussed (Kantrowitz). The instability of a liquid sodium film accelerated by a magnetic field was also described (Colgate).

Some obvious criticisms can be made: as is natural in a report of a symposium, there is some overlapping of sections; on the other hand, often (and particularly in the experimental chapters) too much has been omitted for easy comprehension. Again, the standards aimed at are very different ; for example, most authors eschew mathematics except as a means of illustrating the argument, but the section by Burgers makes few concessions to human frailty. There are also too many rather obvious misprints.

None the less, a general account of this kind will undoubtedly fill a need. The book gives an interesting and readable account of some of the recent work. As a part of this has not been discussed in earlier reports, the book has a definite value.

$$
\text { T. G. Cowling }
$$

\section{RECENT NUCLEAR PHYSICS}

Progress in Nuclear Physics

Vol. 6. Edited by Prof. O. R. Frisch. (Progress Series.) Pp. vii +297. (London and New York : Pergamon Press, 1957.) 84s. net.

THERE are nine articles in Prof. Frisch's sixth volume: on isotope separation by multistage methods, by T. F. Johns; nuclear models, by R. J. Eden; nuclear moments and spins, by K. F. Smith; spectroscopy of mesonic atoms, by M. B. Stearns; masses of atoms of $A>40$, by H. E. Duckworth; electromagnetic isotope separation, by M. L. Smith ; fission recoil, by G. N. Walton; masses of atoms of $A<40$, by J. Mattauch and F. Everling; and parity non-conservation in weak interactions, by $\mathrm{O}$. $R$. Frisch and T. H. R. Skyrme.

Four of the articles are devoted to isotopes. Johns reviews the principles and results of separation by electrolysis, distillation, chemical exchange, diffusion, and thermal diffusion, all of which may be used on a large scale, and refers to other methods used on a laboratory scale. The electromagnetic process, reviewed by M. L. Smith, gives high enrichment in one stage and is much used for gram quantities of $a$ wide range of isotopes. Duckworth tabulates the latest mass values for $A>40$, discussing the sources and the remaining inconsistencies, and proposes mass standards for the heavier atoms. Mattauch and
Everling treat the lighter elements, and review the caloulation of binding energies and masses from the observed data; they direct attention to small but systematic discrepancies between mass spectroscopic and nuclear data.

Many different nuclear models have been developed to represent various aspects of nuclear behaviour, and Eden's theoretical survey deals with the main features of models for low-energy nuclear structure, the compound nucleus model for nuclear reactions, the statistical model, the optical model, models for highenergy reactions, and recent work on a many-body theory. K. F. Smith gives a thorough description of the experimental determination of nuclear spins and magnetic and electric moments, together with the theory underlying the methods and the interpretation of the results. Mrs. Stearns shows how spectroscopic studies of mesonic atoms, made possible by the intense beams from synchrocyclotrons, are providing new information about nuclei (for example, on nuclear radii and charge distribution) and reviews the results obtained.

The important and exciting discovery of parity non-conservation in certain weak interaction phenomena occurred after publication of the previous volume. Frisch and Skyrme now review the experimental and theoretical situations, respectively. The results of experiments on asymmetries in beta-decay and $\pi-\mu-e$ decay have been broadly accounted for by the two-component neutrino theory, though there are some discrepancies. Work in this field is still in its infancy, but Prof. Frisch has wisely broken his normal rule (of including only those subjects which have attained a measure of stability) in view of its very great interest and importance.

In the remaining article, Walton reviews the fission process with emphasis on the recoiling fission fragments and their slowing down in the surrounding matter, including chemical properties and effects.

The material is again authoritatively and clearly presented, with plenty of graphs and tables where appropriate, and all the articles carry good lists of references. Publication has been rather late again, but Prof. Frisch promises greater regularity in future, with volumes appearing about September each year.

T. G. Pickavance

\section{PARASITE AND HOST}

First Symposium on Host Specificity among Parasites of Vertebrates

Pp. 324. (Neuchatel : Institut de Zoologie, Université de Neuchatel, 1957.) n.p.

7 HE complex subject of host-specificity among parasites, so important for the understanding and control of organisms living in this manner, has waited too long for the organized investigation which was started, in April 1957, by the conference the proceedings of which are published in this remarkable volume. Originating, as Prof. J. G. Baer, the general secretary of the conference, explains, in a conversation between Miss Theresa Clay and Prof. Ernst Mayr, the conference was financed by the International Union of Biological Sciences and the University of Neuchâtel, and the results have amply justified the expenditure incurred.

It is impossible to indicate, in a short space, the wealth of information, and of stimulating argument, suggestion and speculation, that the volume contains. Nor would it be fair to select for special mention any 